\title{
Single base station positioning based on multipath parameter clustering in NLOS environment
}

\author{
Yong Wang, Qihong Wu, Mu Zhou, Xiaolong Yang ${ }^{*}$, Wei Nie and Liangbo Xie
}

*Correspondence:
yangxiaolong@cqupt.edu.cn
School of Communication and
Information Engineering,
Chongqing University of Posts and
Telecommunications, Chongqing,
China

*Correspondence: yangxiaolong@cquptedu.cn School of Communication and nformation Engineering China

\begin{abstract}
This paper proposes a scattering area model for processing multipath parameters achieve single base station positioning. First of all, we construct a scattering area model based on the spatial layout of obstacles near the base station and then collect the multipath signals needed for positioning and extract parameters. Second, we use the joint clustering algorithm improved by k-means clustering and mean shift clustering algorithm to process the parameters and extract useful information. Third, the processed information is combined with the spatial layout information of the scattering area model to construct equations, and then the solving problem of equations is converted into a least-squares optimization problem. Finally, the Levenberg-Marquardt (LM) algorithm is used to solve the optimal solution and estimate the mobile target position. The simulation results show that the positioning algorithm in this paper can be used by a single base station to locate the target in an outdoor non-line-of-sight (NLOS) environment, and the accuracy is improved compared with the traditional positioning algorithm.
\end{abstract}

Keywords: Single base station positioning, NLOS, Multipath signal, Parameter clustering, Levenberg-Marquardt algorithm

\section{Introduction}

Outdoor positioning is a practical function in people's daily life, which has been involved in many aspects of life, and it is also one of the basic service and application modules of government and commercial units, even in the military field. Many companies and scientific research teams are engaged in related research [1,2]. At present, the mature outdoor positioning technology is based on satellites, such as the Global Positioning System (GPS) and the BeiDou Navigation Satellite System (BDS). However, some shortcomings exist in the satellite-based positioning system. Firstly, the cost of using the satellite is very expensive. Secondly, at least four satellites are required to get target positions accurately. Thirdly, environmental factors have a great impact on positioning accuracy [3], such as the building or terrain-blocking signal, the extreme weather environment, and geomagnetic storms, all of which may could lead to large positioning errors.

(C) The Author(s). 2021 Open Access This article is licensed under a Creative Commons Attribution 4.0 International License which permits use, sharing, adaptation, distribution and reproduction in any medium or format, as long as you give appropriate credit to the original author(s) and the source, provide a link to the Creative Commons licence, and indicate if changes were made. The images or other third party material in this article are included in the article's Creative Commons licence, unless indicated otherwise in a credit line to the material. If material is not included in the article's Creative Commons licence and your intended use is not permitted by statutory regulation or exceeds the permitted use, you will need to obtain permission directly from the copyright holder. To view a copy of this licence, visit http://creativecommons.org/licenses/by/4.0/. 
The outdoor positioning technology based on a single communication base station (BS) is cost-efficient and convenient for users [4]. It only needs to extract the parameter information from the electromagnetic wave signal of the mobile terminal in the communication process to operate. In 1996, the Federal Communications Commission (FCC) issue the Enhanced 911 (E911) [5], which claim that the wireless cellular networks must be able to provide the location-based service (LBS) to mobile terminals. As a result, the positioning technology based on cellular BSs has been the focus of research. In the cellular BS-based positioning system, the signal parameters from the mobile terminal are extracted for positioning. The commonly used measurement parameters include the time of arrival (TOA), time of flight (TOF), angle of arrival (AOA), time difference of arrival (TDOA), and received signal strength (RSS) [6, 7]. By considering the accuracy of signal measurement and parameter extraction, the research early is often based on single parameters. Generally, positioning systems based on single parameters often need multiple BSs to coordinate location, such as the Chan algorithm [8] and the Caffery algorithm [9]. However, if multiple BSs are used, we must consider the synchronization between BSs, the pressure of signal transmission on the network, and the cost and management of hardware equipment. Moreover, to ensure the quality of service (QoS), the mobile terminal will only connect to the service BS in the wireless communication system, then multi-station cooperation will have the problem that the signal can be detected effectively at the same time or not [10-12]. Therefore, in many studies, the positioning algorithm based on multi-parameter fusion is proposed to reduce the number of cooperative cellular BSs [13]. With multi-input multi-output (MIMO) technology [14] and the smart antenna technology $[15,16]$, the realization of positioning technology based on parameter fusion becomes possible.

Compared with the multi-station, the single-station positioning algorithm has obvious advantages in equipment cost and complexity, response time, mobility, and flexibility. Therefore, the single station positioning system has high research value in the current era of advocating energy saving and high efficiency. The common single-station positioning system relies on TOA/AOA hybrid positioning, as shown in Fig. 1, which requires the line-of-sight (LOS) path and sufficiently accurate measurement parameters. However, in the outdoor environment with dense obstacles, the LOS path for the mobile terminal is often blocked, and the signal propagates through other paths to the BS in the form of the multi-path, which is called as the non-line-of-sight (NLOS) propagation. If it is mistakenly recognized as the LOS propagation, the accuracy of the positioning system will greatly deteriorate. The positioning technology based on the multi-station mainly depends on suppressing the error caused by the NLOS and restoring LOS information, such as the classical Wylie algorithm [17]. In the single-station positioning system, the BS lacks other BSs to provide cooperative information, so it is difficult to conduct positioning under the condition of the NLOS. Fortunately, if combined with the advantages of the single station mentioned above, the single-station NLOS positioning technology is promising.

The single BS positioning system in the NLOS environment often has the problem of insufficient location information because of the lack of cooperation of other BSs. The obstacles hindering the propagation of the LOS and the multipath signals generated by it can also provide information for positioning. By making full use of the information combined with mathematical statistics, machine learning and intelligent optimization can operate and improve positioning accuracy [18]. The authors in [19] use support vector 


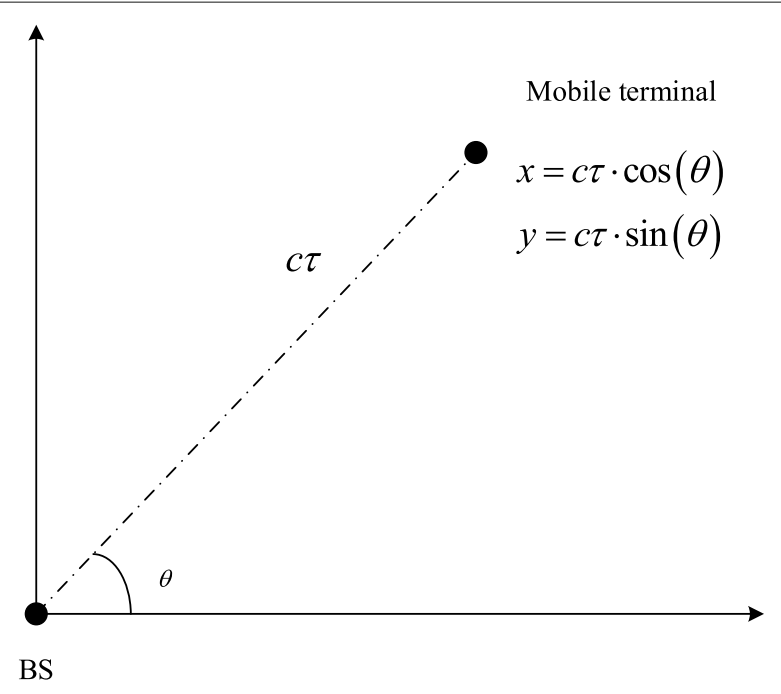

Fig. 1 Traditional single station positioning scheme

machine (SVM) to realize single station positioning in the MIMO system, but it requires the Angel of Departure (AOD) of signal. For the mobile terminals, they are difficult to measure this parameter. In the research of the single-site positioning technology in the NLOS environment, the idea is to make use of the information of scatterers. A deterministic propagation model is used in [20]. In this scheme, the environmental information around the BS is sampled, the location of the scattering point is found according to the AOA of the multipath signal, and then the equation is established with the TOA to determine the location of the mobile terminal. However, this scheme requires $360^{\circ}$ omnidirectional sampling of the environment near the BS. Therefore, the scattering model is often introduced in the single BS positioning system in the NLOS environment for analysis [21]. Common scattering models include the Ring of Scattering (ROS), Disk of Scattering (DOS), and Gaussian Scattering Density Model (GSDM) [22, 23]. The authors in [24] quote ROS and DOS models and use the idea of reconstructing LOS paths to conduct positioning. This scheme analyzes the probability density function of TOA measurements of two models, and takes the TOA of the LOS path as a parameter of the density function, and then reconstructs the TOA of the LOS path by Bayesian and maximum likelihood estimation. However, the algorithm of this scheme is complex, and the calculation process is also complicated. Based on the scattering point model, a pseudo-target dynamic feasible region constraint method is proposed in [25]. This algorithm extracts AOA and TOA of the multipath signal to recover multiple pseudo-targets, and combines the scattering point model to determine the feasible region of the real target from the pseudo-target, and then estimates the position of the target. However, the scheme in [25] requires the maneuverability of the receiver and it is not suitable for BSs in cellular networks.

In order to solve the above problems, a scattering area model with the specific spatial layout information is proposed in this paper. First of all, in this model, the measured parameters of the multipath signal are extracted by the BS, and then the parameters are clustered by the improved joint clustering algorithm. Second, the geometric equations are constructed according to the spatial layout information in the scattering area model and 
the system of equations established in this scheme can eliminate the clock synchronization error. Finally, the position of the target terminal is estimated by solving the equations with the Levenberg-Marquardt (LM) algorithm. This study is simulated by the Matlab and the Wireless Insite, and the results show that the scheme can achieve outdoor NLOS single station positioning and improve positioning accuracy.

The rest of this paper is organized as follows. Section 2 presents the positioning scheme, including the description of the model, clustering algorithm, and target position calculation. Section 3 presents the result of simulation, describing the influence of different factors on the positioning results and use the Wireless Insite to simulate the real environment to verify the positioning algorithm. Finally, Section 4 reports the conclusions.

\section{Methods}

\subsection{Scattering model}

\subsubsection{Common scattering model}

In previous studies, a variety of scattering models have been proposed, among which the most commonly used models are ROS and DOS, as shown in Fig. 2. The ROS regards the scattering points to be randomly distributed on the circle with the target as the center and satisfies a certain numerical radius, and the DOS regards the scattering points as obeying the two-dimensional Gaussian random distribution in the circle. In the traditional scheme, the multipath is introduced, the system of equations adds an unknown parameter, and too many unknown parameter will make the solving process of the system complicated.

\subsubsection{Scattering area model}

Aiming at the problem of the insufficient information in the traditional NLOS singlestation positioning system, this paper proposes a new signal reflection model in the NLOS environment. In the case of dense obstacles in the environment, there are often areas with reflected signals near the fixed BSs, such as tall or dense buildings. The propagation signal from the terminal is mainly reflected in these areas, and the scattering points are mainly distributed in these areas. In order to facilitate analysis, the design of the scattering area model in this paper is as follows. Referring to the spatial layout of the environment near the BS, the scattering area is set as a circular area with a certain point as the center and a
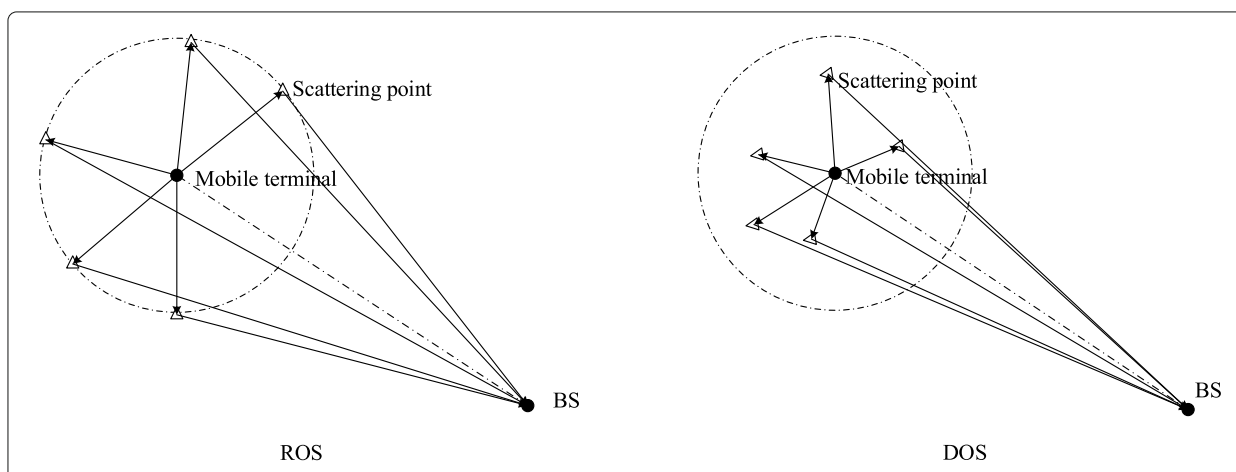

Fig. 2 ROS and DOS 
fixed radius. The center of the circle is regarded as the center of the scattering area and the scattering point is in the scattering area. The area obeys a two-dimensional Gaussian random distribution, and the average value is the center coordinate of the scattering area. The signal entering the scattering area is reflected by these scattering points and received by the BS. The scattering area model is shown in Fig. 3.

The scattering area model is shown in Fig. 3. Each blue solid circle is the center of a scattering area $\left(x_{i}, y_{i}\right), i=1,2, \ldots, N$, where $N$ is the number of scattering areas, and each dotted circle is a defined scattering area with the radius is $r_{i}$. The scattering point is in the circle, which obeys the two-dimensional Gaussian distribution with the mean value of the coordinate value of the scattering area center. Actually, scattering points that can reflect signals are not only limited in the defined scattering area but also exist far away from the scattering area. In this paper, they are called as interference scattering points, which are shown as red triangle points in Fig. 3. The signal transmitted by the target is reflected by the scattering point and received by the BS. Letting $n$ be the number of measured multipaths, coordinates of scattering points are $\left(x_{s j}, y_{s j}\right), j=1,2, \ldots, n$, and $\left(x_{B}, y_{B}\right)$ is the coordinate of the BS.

\subsubsection{Measurement parameters}

In this scheme, the TOA and the AOA are extracted from the channel state information (CSI). Under the condition of the NLOS propagation, the TOA of the signal is the flight time that the signal is transmitted from the terminal to the scattering point, and then from the scattering point to the BS. The AOA is the relative angle between the scattering point of the scattering point and the BS. In the $4 \mathrm{G}$ or $5 \mathrm{G}$ wireless communication systems, the wireless signals relies on the orthogonal frequency division multiplexing (OFDM) technology to divide the system frequency band into several separate sub-carriers. The CSI on the subcarrier contains the relevant parameters of the carrier signal, and the information needed for positioning can be extracted by using the super-resolution parameter estimation technology. The scheme proposed in this paper works in the NLOS environment, that is, the environment with dense obstacles without the LOS.

The AOA and the TOA of the multipath signal arriving at the BS are represented as $\left(\theta_{j}, \tau_{j}\right), j=1,2, \ldots, n$, where $\theta_{J}$ is the AOA signal and $\tau_{j}$ is the TOA of the multipath, which

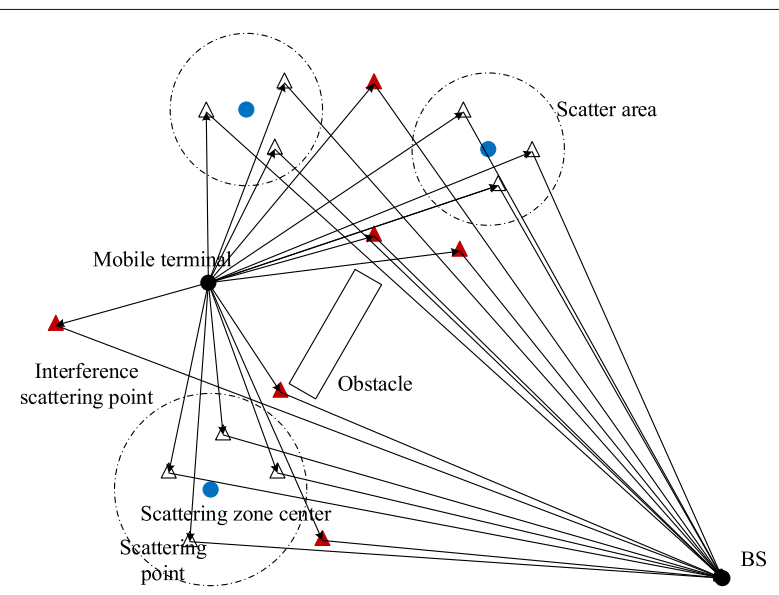

Fig. 3 Scattering area model 
are received at the BS. Generally, the propagation speed of the electromagnetic waves is constant, denoted as $c$, and then the relationship between the measured parameter and the scattering point is expressed as

$$
\left\{\begin{array}{l}
\theta_{j}=\arctan \left(\frac{y_{s j}-y_{B}}{x_{s j}-x_{B}}\right) \\
c \tau_{j}=c \tau^{\prime}{ }_{j}+\sqrt{\left(x_{s j}-x_{B}\right)^{2}+\left(y_{s j}-y_{B}\right)^{2}}
\end{array}\right.
$$

where $\tau_{j}^{\prime}$ is the time for the signal propagating from the target terminal to the $j$ th scattering point.

\subsection{Joint clustering algorithm}

According to the model constructed in this paper, the scattering points in the same scattering area have the characteristic of aggregation, by which the AOA and the TOA have the similar distribution in the two-dimensional space, so the clustering algorithm can be used to process these parameters. The clustering algorithm is a type of unsupervised learning algorithms, which can be used to classify data without labels. We let $\left(\theta_{j}, \tau_{j}\right)$ be the measurement parameter of the BS and the sample used for clustering be $S=\left\{s_{1}, s_{2}, \cdots, s_{n}\right\}$, where $s_{j}$ is the sample parameter, as shown in (2).

$$
s_{i}=\left\{\left(\alpha_{i}, \beta_{i}\right) \mid \alpha_{i}=c \tau_{i} \cdot \cos \left(\theta_{i}\right), \beta_{i}=c \tau_{i} \cdot \sin \left(\theta_{i}\right)\right\}
$$

Here, $s_{j}$ is also called as the pseudo target coordinate value determined by the $j$ th multipath measurement parameter, so each scattering point $\left(x_{s j}, y_{s j}\right)$ corresponds to a sample parameter $s_{j}$.

The scattering point $\left(x_{s j}^{i}, y_{s j}^{i}\right)$ in the scattering area $i$ is regarded as obeying the expected Gaussian distribution with the center of the scattering area $\left(x_{i}, y_{i}\right)$. If there are enough scattering points, the mean value of the scattering point coordinates in a scattering area approximately equals to the center of the scattering area $\left(x_{i}, y_{i}\right)$. Then, the average value of the pseudo-target coordinate values corresponding to all the scattering points in the scattering area $i$ is approximately equal to the pseudo-target coordinate value formed by the signal reflected by the center of the scattering area $\left(x_{i}, y_{i}\right)$. If the average value after clustering is approximated as the pseudo-target coordinate value of the signal reflected from the center of the scattering area $\left(x_{i}, y_{i}\right)$, and the corresponding AOA and reach distance are calculated by using the average value of the clustering, a set of equations can be established to conduct positioning.

At present, there are many clustering algorithms in the existing research. Among them, the k-means clustering algorithm is based on the Euclidean distance between the data to calculate the similarity, so the operation is simple, and the time complexity tends to be linear in general [26]. However, the result of the k-means clustering algorithm is easily affected by the noise and the isolated sample points. The result of k-means clustering is shown in Fig. 4. It can be seen that the outlier participating in clustering has a great influence on the clustering result. The above problem can be solved by using the mean shift clustering algorithm [27], which is based on the data distribution density to measure the similarity. The interference points are scattered and deviate from the main scattering area. The algorithm will separate them into clusters. However, the sliding window size of mean shift clustering has an important influence on clustering performance. In this paper, the window size is related to the radius of the scattering area. If all data points are clustered once, the window size of mean shift clustering should be set based on the 


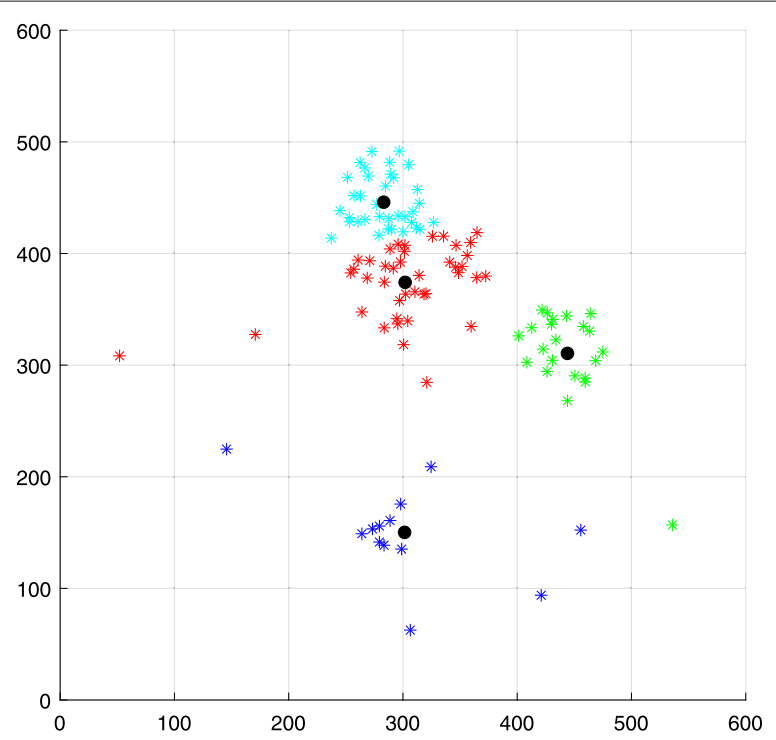

Fig. 4 K-means clustering result

maximum scattering area, but the clustering performance of the clusters corresponding to the scattering area with a smaller radius is poor. The results of mean shift clustering are shown in Fig. 5, in which the dashed circle represents the size of the clustering window. The fixed-size sliding window does not have a good clustering effect when the difference in the radius of the scattering area is too large, and some points are even not classified into the cluster.

In this paper, two clustering algorithms are combined into a joint clustering algorithm. First of all, all the data are roughly distinguished by using the k-means clustering, and then the mean shift clustering is used to filter out the interference points for the result obtained from k-means clustering. After k-means clustering, we can get the parametric

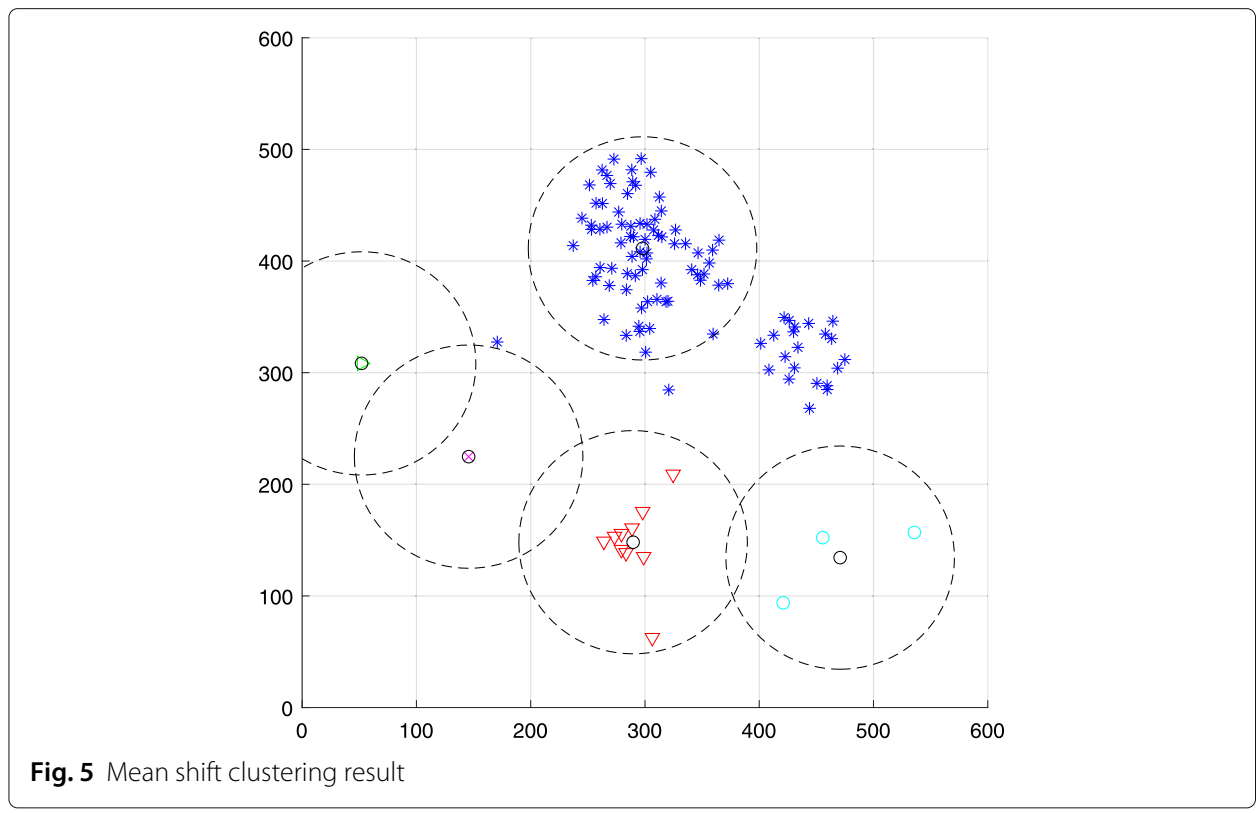


clustering results $\left(\alpha^{\prime}{ }_{i}, \beta_{i}^{\prime}\right)$. Each result corresponds to a scattering area, the center of the scattering area is $\left(x_{i}, y_{i}\right)$, and its corresponding radius is $r_{i}$. For each k-means clustering result, the equation for the sliding window size of the mean shift clustering is:

$$
w_{i}=r_{i} \cdot \frac{2 \sqrt{\left(\bar{\alpha}_{i}-x_{B}\right)^{2}+\left(\bar{\beta}_{i}-y_{B}\right)^{2}}}{\sqrt{\left(x_{i}-x_{B}\right)^{2}+\left(y_{i}-y_{B}\right)^{2}}}
$$

The clustering result of the joint clustering algorithm is shown in Fig. 6. The size of the dotted circle represents the sliding window size of the mean shift cluster in each $\mathrm{k}$-means clustering result, and each dotted circle represents a cluster. In Fig. 6, the clusters with a large number of distributed points represent the clustering results of the pseudo-target corresponding to the reflection signals of the scattered points in the scattering area, and the clusters with fewer distributed points represent the clustering result of the pseudotarget corresponding to the interference scattering points. The clustering results with the largest number of pseudo-target points are selected as references, and the clustering center is used as a parameter to establish an equation set, whereas other clustering results are discarded as the corresponding results of interference scattering points.

\subsection{Target position calculation}

The information that we have got involve the center position of scattering areas $\left(x_{i}, y_{i}\right)$, $i=0,1, \ldots, N$ and the clustering result obtained by the joint clustering algorithm $\left(\bar{\alpha}_{i}, \bar{\beta}_{i}\right)$. The corresponding parameter $\left(\bar{\theta}_{i}, \bar{\tau}_{i}\right)$ is deduced from (1), and then the AOA between the center of the scattering area $\left(x_{i}, y_{i}\right)$ and the BS is calculated as

$$
\theta_{i}^{\prime}=\arctan \left(\frac{y_{i}}{x_{i}}\right) \quad i=0,1, \ldots, N
$$

The parameters $\bar{\theta}_{i}$ and $\theta_{i}^{\prime}$ are matched according to the principle of the minimum difference, and $\left(\bar{\theta}_{i}, \bar{\tau}_{i}\right)$ is taken as the approximate measurement parameter with the scattering

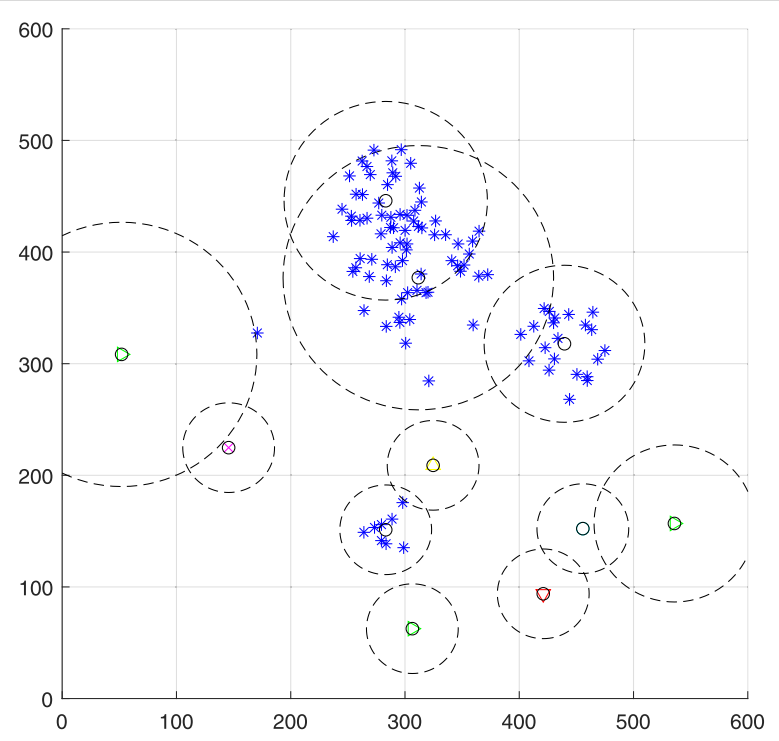

Fig. 6 Joint clustering result 
center $\left(x_{i}, y_{i}\right)$. If the coordinate of the target terminal is $(x, y)$, the relations of the target, the scattering point, and the BS are shown in Fig. 7.

According to the geometric structure in Fig. 7, we can establish

$$
\left\{\begin{array}{c}
\sqrt{\left(x-x_{1}\right)^{2}+\left(y-y_{1}\right)^{2}}=c \bar{\tau}_{1}-\sqrt{\left(x_{1}-x_{B}\right)^{2}+\left(y_{1}-y_{B}\right)^{2}} \\
\sqrt{\left(x-x_{2}\right)^{2}+\left(y-y_{2}\right)^{2}}=c \bar{\tau}_{2}-\sqrt{\left(x_{2}-x_{B}\right)^{2}+\left(y_{2}-y_{B}\right)^{2}} \\
\vdots \\
\sqrt{\left(x-x_{N}\right)^{2}+\left(y-y_{N}\right)^{2}}=c \bar{\tau}_{N}-\sqrt{\left(x_{N}-x_{B}\right)^{2}+\left(y_{N}-y_{B}\right)^{2}}
\end{array}\right.
$$

In the actual communication process between the target terminal and the BS, the parameters have the synchronization error due to the out of sync of clocks between the transmitter and the receiver, that is

$$
\tau_{T O A}=\tau_{\text {TOA_true }}+\tau_{s_{-} \text {err }}+\tau_{n}
$$

where $\tau_{T O A_{-} \text {true }}$ is the true TOA, $\tau_{s_{-} \text {err }}$ is the delay of the synchronization error, and $\tau_{n}$ is the measurement error caused by the white noise. In the single station positioning system, the clock synchronization error of each multipath is the same in the same signal transmission process, so the difference equation of two multipath TOAs is established to eliminate the synchronization error. By assuming that the distance from the target to the scattering point is $l_{i}(i=0,1, \cdots, N)$, we have

$$
l_{i}=c \bar{\tau}_{i}-\sqrt{\left(x_{i}-x_{B}\right)^{2}+\left(y_{i}-y_{B}\right)^{2}}
$$

If the number of scattering areas is $N$, the $N-1$ equations can be established as

$$
\left\{\begin{array}{c}
\sqrt{\left(x-x_{N}\right)^{2}+\left(y-y_{N}\right)^{2}}-\sqrt{\left(x-x_{1}\right)^{2}+\left(y-y_{1}\right)^{2}}=l_{N}-l_{1} \\
\sqrt{\left(x-x_{N}\right)^{2}+\left(y-y_{N}\right)^{2}}-\sqrt{\left(x-x_{2}\right)^{2}+\left(y-y_{2}\right)^{2}}=l_{N}-l_{2} \\
\vdots \\
\sqrt{\left(x-x_{N}\right)^{2}+\left(y-y_{N}\right)^{2}}-\sqrt{\left(x-x_{N-1}\right)^{2}+\left(y-y_{N-1}\right)^{2}}=l_{N}-l_{N-1}
\end{array}\right.
$$

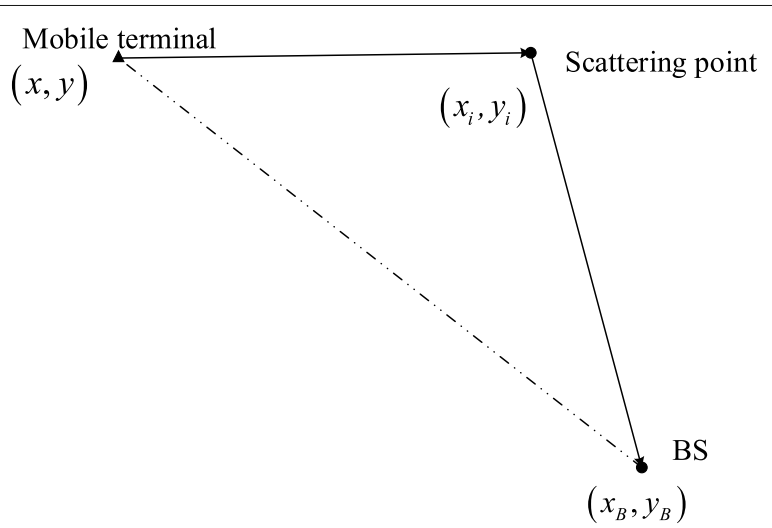

Fig. 7 Single station positioning structure in the NLOS 
In practice, the (8) is not exactly equal. The solving of deterministic equation can be transformed into the solving of optimization problem. First, we rewrite (8) as

$$
\begin{aligned}
\varphi_{i}(x, y) & =\sqrt{\left(x-x_{N}\right)^{2}+\left(y-y_{N}\right)^{2}}-\sqrt{\left(x-x_{i}\right)^{2}+\left(y-y_{i}\right)^{2}}-\left(l_{N}-l_{i}\right), \\
i & =0,1, \cdots, N-1
\end{aligned}
$$

where $\varphi_{i}(x, y)$ is the error objective function. Then, the solution that minimizes the objective function shown in (10) is the position of the target.

$$
\varepsilon(x, y)=\frac{1}{2} \sum_{i=1}^{N-1} \varphi_{i}^{2}(x, y)
$$

This paper uses the LM algorithm to solve (10). The LM algorithm is an iterative algorithm for finding the extreme value of a function, which can be used to solve the nonlinear least squares problem. The positioning scheme in this paper introduces the spatial layout as an information supplement and uses a clustering algorithm to process multipath parameters, which can greatly simplify equations and avoid the situation that the function does not converge in the limited domain. In the LM algorithm, the solution that minimizes the objective error function is the optimal solution, which is the estimation of the target terminal position.

\section{Results and discussion}

The positioning range is $300 \mathrm{~m}$ by $300 \mathrm{~m}$, and the coordinate of the BS is $(0,0)$. The parameters of the AOA and the TOA are generated by the simulation of the scattering area model. With the known central coordinates and radius of the scattering area, a certain number of random points are distributed in each scattering area. These random points obey the two-dimensional Gaussian distribution with the mean value of the coordinate value of the scattering area center, and the random points within the radius of the scattering area are selected as scattering points. In addition, in order to test the performance of interference scattering points outside the scattering area, several interference scattering points are randomly generated in the positioning range. The TOA is the sum of the flight time of the signal from the mobile terminal to the scattering point and the flight time from the scattering point to the BS, and the flight distance of the signal is the TOA multiplied by $c$. The relative angle between scattering points and the BS represents the simulated AOA. Two hundred independent experiments are conducted in each round of simulation experiments.

\subsection{Influence of variables}

The variables that affect the positioning results include the number of scattering areas, the size of scattering areas, the number of random points, the number of interference scattering points, measurement error of AOA, and measurement error of TOA. The influence of these variables on the positioning results will be discussed in the following.

Figure 8 shows the effect of the number and size of scattering areas on the result. All scattering areas are set with the same radius during the simulation. It can be seen from the figure that when the number of scattering areas is less than 5 , the positioning accuracy increases with the increase of the number of scattering areas. However, when the number of scattering areas increase to 6 , the positioning accuracy is decreases. Too many scattering areas and scattering points lose their clustering characteristics, resulting in a 


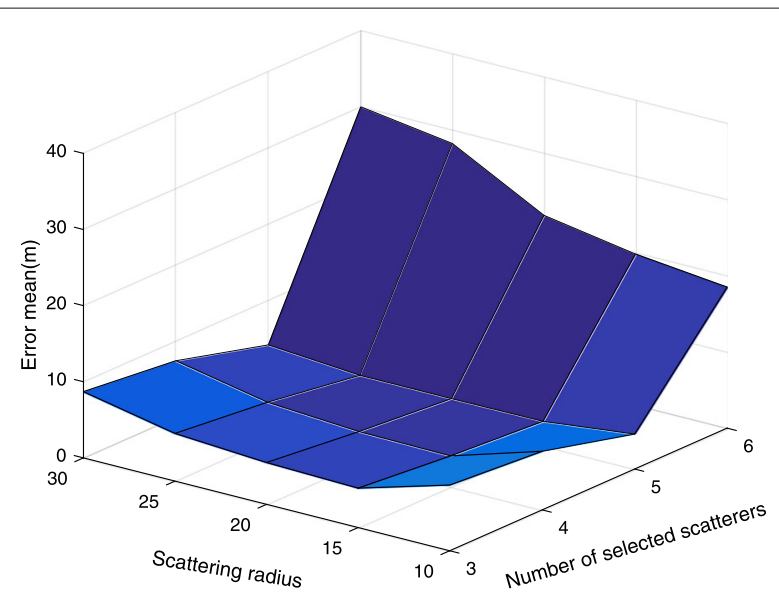

Fig. 8 Influence of the number and size of scattering areas on positioning performance

decrease in clustering effect. When the radius is less than $20 \mathrm{~m}$, the larger the radius of the scattering area, the smaller the positioning error. When the radius is greater than 20 $\mathrm{m}$, the scattering points will lose the clustering characteristics, resulting in a decrease in the clustering effect.

It can be seen from the above analysis that the structure of the scattering area has an important influence on the positioning result. The method of determining the scattering area is as follows. We examine the spatial layout of the main reflection signal area near the BS, such as the location of the building group, initially determine the location of the scattering area, and then combine the multipath signal parameters measured by the BS to determine the specific scattering area. After that, we optimize each area into a circular scattering area and use the center of the circle as the center of the scattering area. By considering the environment, when selecting the scattering area, it should be ensured that the scattering area covers a wide area, and there is still enough space between the scattering areas.

Figure 9 shows the effect of the number of random points and interference scattering points on positioning accuracy. The number of multipaths equals to the number of

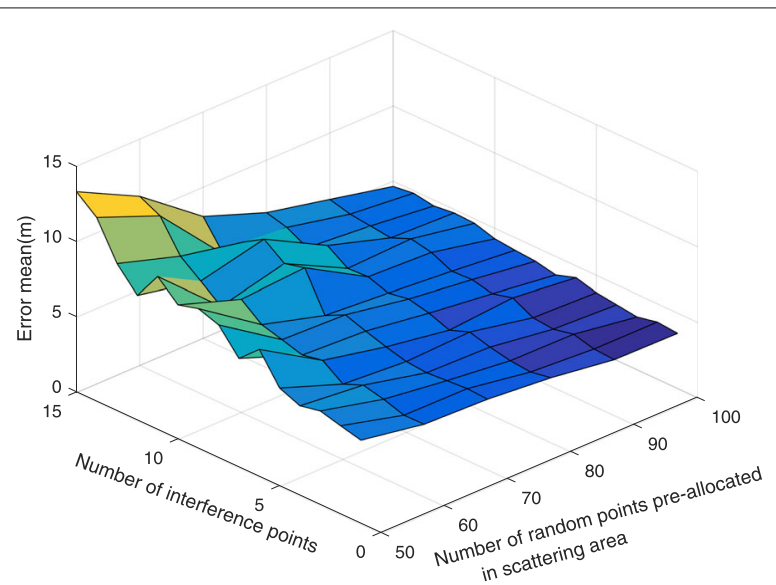

Fig. 9 Influence of interference points and pre-assigned random points on the result 
scattering points. In the simulation process, the more random points preset for the scattering area, the more scattering points are distributed within the radius of the scattering area. Obviously, the more random points, that is, the more multipaths extracted by the $\mathrm{BS}$, the higher the positioning accuracy. When no interference point is introduced, the sample parameters are corresponding to the scattering points in the scattering area, and the results after clustering the sample parameters correspond to the respective scattering areas, the parameter $\left(\bar{\theta}_{i}, \bar{\tau}_{i}\right)$ calculated by the clustering center is approximately estimated as the parameter taking the center of the scattering area $\left(x_{i}, y_{i}\right)$ as the scattering point. Thus, when there is no interference scattering point, the positioning result has the error, but the error is small, generally within $5 \mathrm{~m}$. As the number of interference scattering points increases, the mean value and mean square error of the error show an upward trend. However, when the number of random points pre-allocated more than 80 , the number of interference points has little effect on the mean error. Therefore, the joint clustering algorithm can effectively reduce the influence of interference scattering points on positioning accuracy when enough multipaths are extracted.

In the process of simulation, the white noise error is added to the AOA and the TOA, the mean value of the noise is 0 , and the noise intensity is measured by the standard deviation of the error. Since the propagation velocity of electromagnetic wave is always $c$, the TOA error can be measured directly by the measured flight distance error. Figure 10 shows the influence of the measurement parameter error on positioning accuracy, from which we can find the greater the noise standard deviation, the greater parameter accuracy affected by the noise.

\subsection{Influence of clustering}

K-means clustering, mean shift clustering, and joint clustering algorithms are used to cluster the parameters and realize positioning. The simulation results are shown in Fig. 11. In the fixed scene, the positioning result of the mean shift clustering algorithm is better than that of the k-means clustering algorithm, and the positioning result of the joint clustering algorithm is slightly better than using the mean shift clustering algorithm.

According to the previous analysis, compared to using mean shift clustering, the advantage of joint clustering is that it is applicable to a variety of radius sizes of the scattering

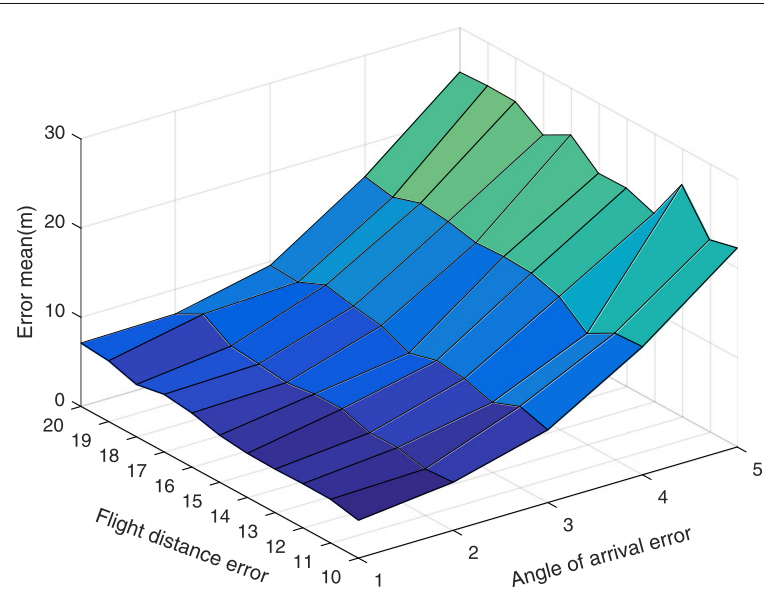

Fig. 10 Influence of parameter measurement error on the result 


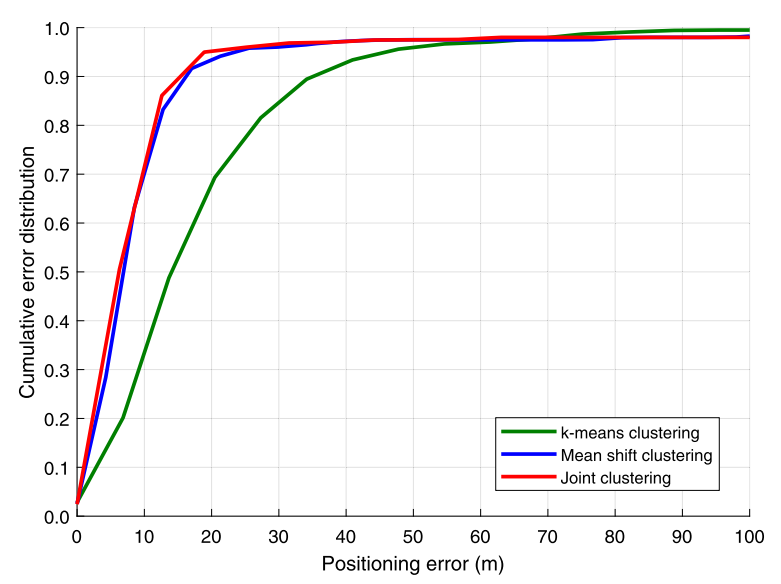

Fig. 11 Results of different clustering algorithms

area. In order to verify this feature of joint clustering, when comparing the positioning results of using mean shift clustering and using joint clustering,the radius of each scattering area takes a random value in $(0,40]$, and we repeat 200 independent experiments. The simulation results are shown in Fig. 12. At this time, the positioning effect based on joint clustering is better than the positioning effect based on mean shift clustering, since the joint clustering algorithm still has a good clustering result in the case of great difference in the radius of the scattering area. Therefore, the joint clustering algorithm is more suitable for the model proposed in this paper.

\subsection{Comparison of different schemes}

The result of the proposed scheme is compared with the ones by using ROS and DOS models, and the results are shown in Fig. 13. The positioning effect of the model scheme in this paper is better than both the ROS model and the DOS model. The positioning error based on the ROS model is smaller than that based on the DOS model, the set-

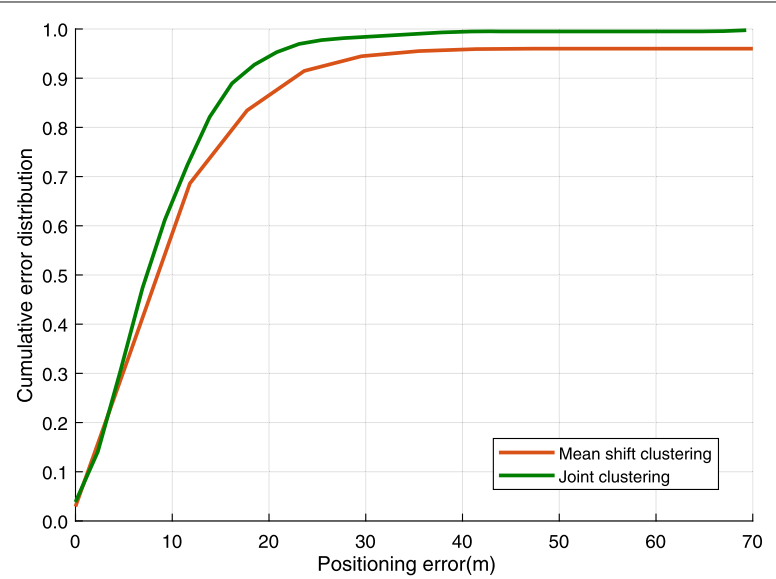

Fig. 12 Error of CDF based on joint clustering and mean shift clustering when the scattering radius is set randomly 


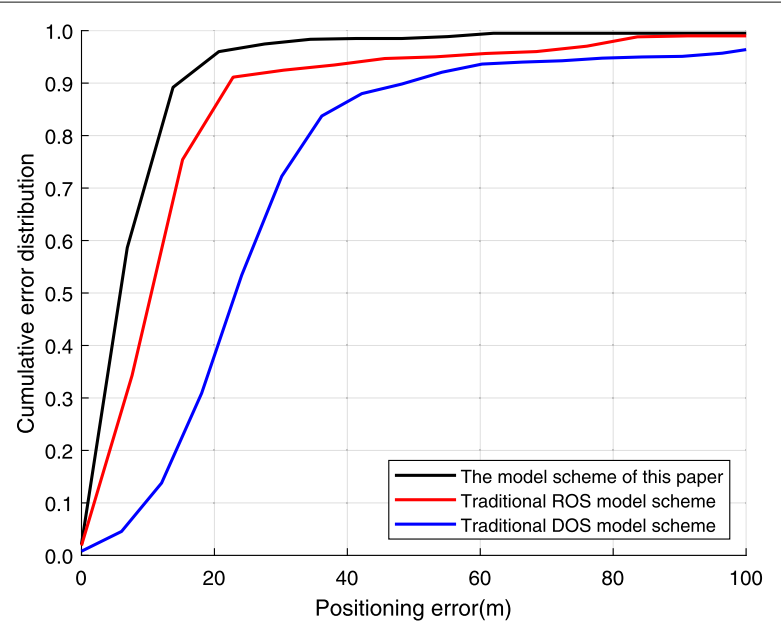

Fig. 13 Error of CDF using different schemes

ting of the scattering points of the ROS model on the ring can not be fully verified in reality, and it is difficult to determine the radius of the ring in the complex environment. Compared with the ROS model, the DOS model is more appropriate to describe the complex environment, but compared with the scheme of this paper, the introduction of the spatial layout information is still insufficient. The simulation results show that the positioning result of the scheme in this paper is obviously better compared to the DOS model.

\subsection{Wireless Insite simulation data}

Wireless Insite is a set of simulation software using the Ray Tracing (RT) model, which can be used to analyze the working characteristics of radio wave transmission and wireless communication system in individual scenes. It can simulate and predict the electromagnetic wave transmission path and the working characteristics of each channel of the communication system in the complex large geographical areas, urban areas, relatively small indoor environments, and mixed environments. In this paper, we use the Wireless Insite software to simulate the actual environment and generate the corresponding data to verify the scheme of this paper.

First of all, the actual scene model is constructed by using the Wireless Insite, based on which the scene environment between the Digital Library in the Chongqing University of Posts and Telecommunications (CQUPT) and the Xinke Building is constructed. As shown in Figs. 14 and 15, the building in the picture is set according to the building structure in the actual scene, the model material is set as the cement concrete, the BS is arranged according to the actual planning, and the yellow dot in Fig. 15 is the location of the BS (only one BS is used at a time). The position of the blue point is the position set by the terminal target, and these positions are consistent with the NLOS condition in the process of simulation.

By taking the target location downstairs of the Xinke Building and the BS on the library in the CQUPT as an example, the simulation ray is shown in Fig. 16.

According to the environmental layout characteristics of the scene area and the relevant information of signal rays, the scattering area is determined. As can be seen from 


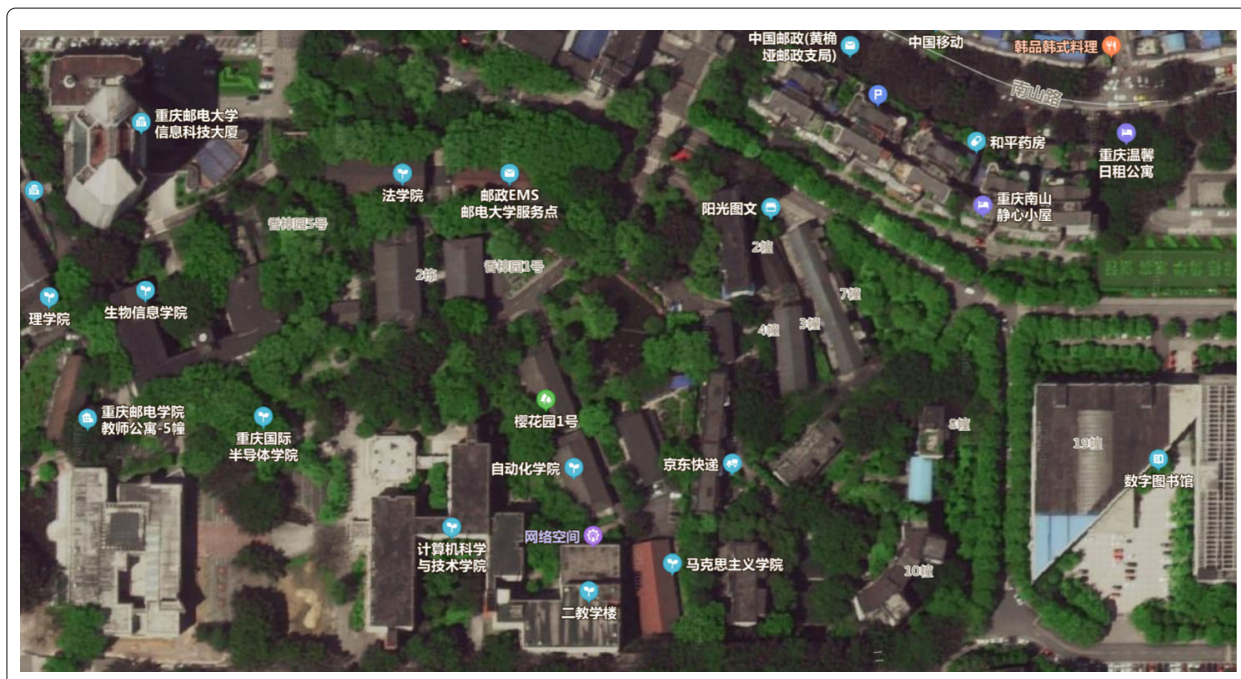

Fig. 14 Satellite scan image of simulation scene

Fig. 16, in this environment, the scattering area mainly depends on the selection of the location of the building. The green square in the figure represents an area where the terminal is located with the range of $5 \mathrm{~m}$ by $5 \mathrm{~m}$, a sampling point is taken with the interval of $1 \mathrm{~m}$, and 25 sampling points are taken for simulation verification, as shown in Fig. 17.

Multiple regions are sampled, the error results are counted, and the cumulative distribution function (CDF) diagram is shown in Fig. 18. In this scheme, $65 \%$ of the error obtained by using the simulation data of Wireless Insite software can be controlled within $21 \mathrm{~m}$.

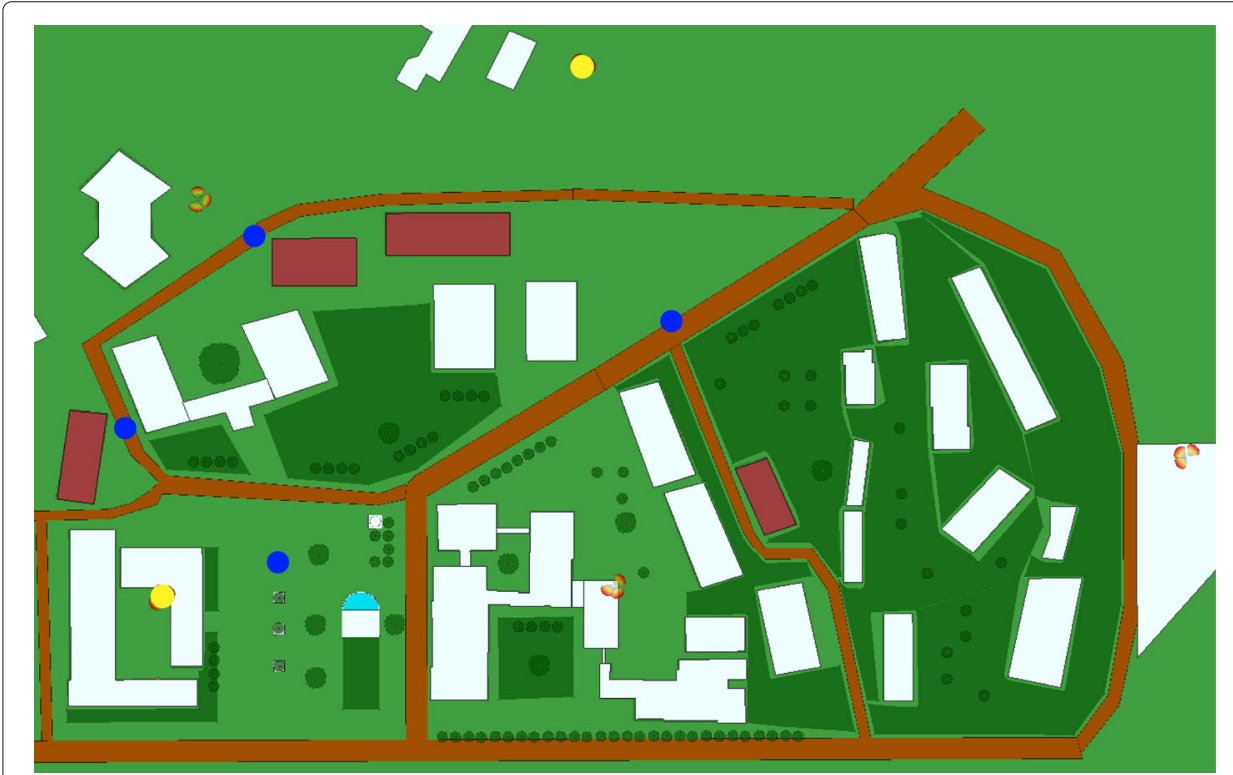

Fig. 15 Map model built by the Wireless Insite 


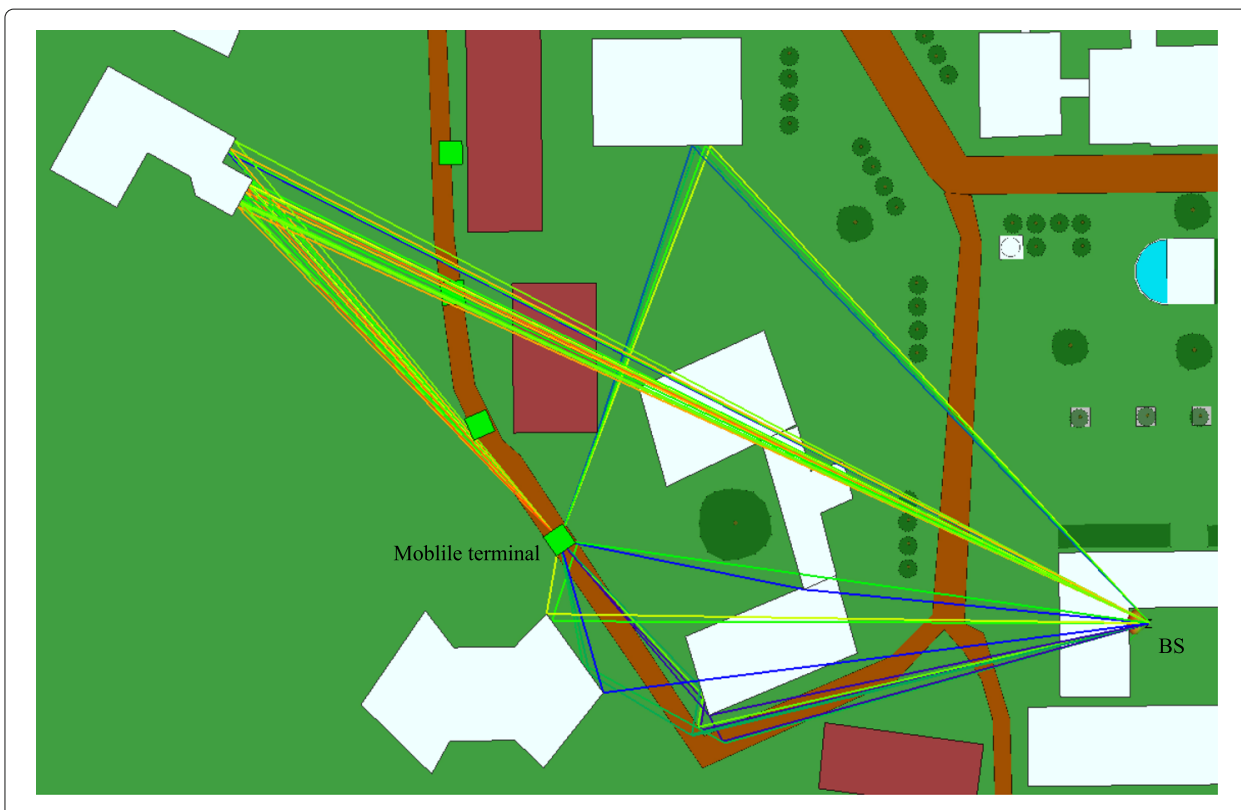

Fig. 16 Simulation ray by the Wireless Insite

\section{Conclusion}

This paper first proposed a novel scattering area model for the actual scene. Then, a single BS positioning algorithm of outdoor NLOS based on parameter clustering was proposed. The positioning algorithm introduced the spatial layout in the environment near the BS as information supplement and made full use of the multipath information. The established positioning equations were simple, and the convergence speed was very fast. In this paper, a joint clustering algorithm was designed to optimized the clustering result. Verified by simulation, the proposed algorithm had better performance, less computation and higher positioning accuracy in outdoor NLOS environment with single BS.

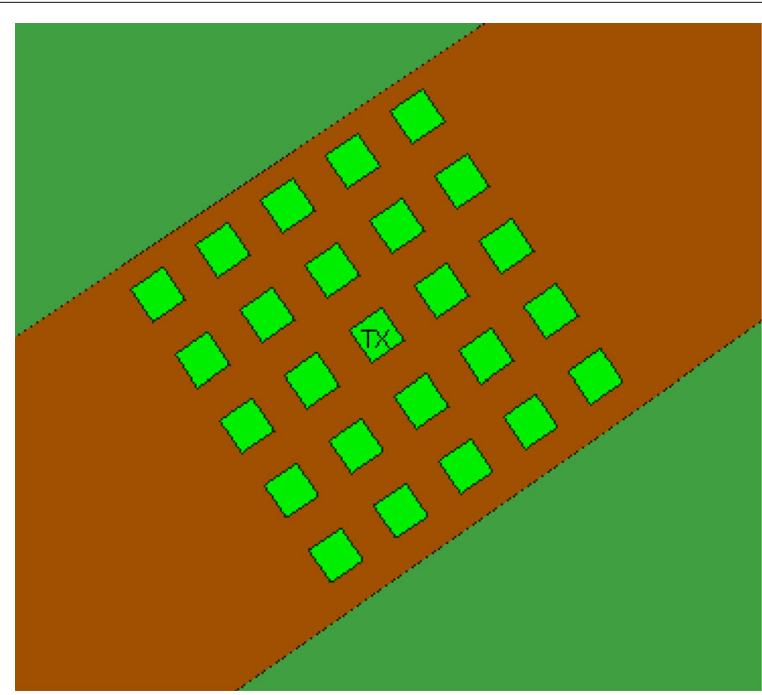

Fig. 17 Schematic diagram of sampling points 


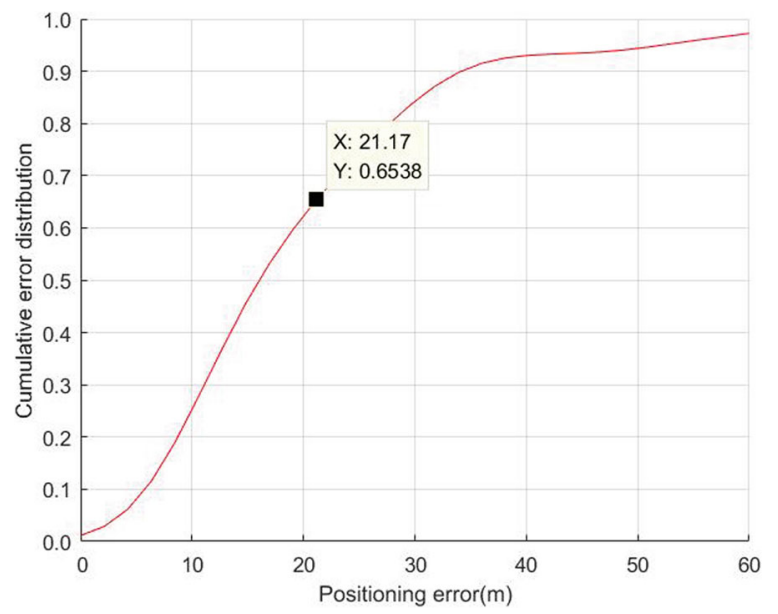

Fig. 18 Wireless Insite simulation results

\section{Abbreviations}

GPS: Global positioning system; BDS: BeiDou navigation satellite system; BS: Base station; FCC: Federal Communications Commission; E911: Enhanced 911; LBS: Location-based service; TOA: Time of arrival; TOF: Time of arrival; AOA: Angle of arrival; TDOA: Time difference of arrival; RSS: Received signal strength; QoS: Quality of service; MIMO: Multi-input multi-output; LOS: Line-of-sight; NLOS: Non-line-of-sight; SVM: Support vector machine; AOD: Angel of Departure; ROS: Ring of Scattering; DOS: Disk of Scattering; GSDM: Gaussian Scattering Density Model; LM: Levenberg-Marquardt; OFDM: Orthogonal frequency division multiplexing; RT: Ray Tracing; CQUPT: Chongqing University of Posts and Telecommunications; CDF: Cumulative distribution function

\section{Acknowledgements}

This work was supported by the School of Communication and Information Engineering, Chongqing University of Posts and Telecommunications.

\section{Authors' contributions}

The algorithms proposed in this paper have been conceived by Y. Wang, Q. Wu, and M. Zhou. Y. Wang, Q. Wu, and X. Yang designed and performed the experiments and wrote the paper. M. Zhou, W. Nie and L. Xie investigated, validated, and revised this paper. The authors approved the final manuscript.

\section{Authors' information}

Yong Wang, School of Communication and Information Engineering, Chongqing University of Posts and Telecommunications, Chongqing, China.

Qihong Wu, School of Communication and Information Engineering, Chongqing University of Posts and Telecommunications, Chongqing, China.

Mu Zhou, School of Communication and Information Engineering, Chongqing University of Posts and Telecommunications, Chongqing, China.

Xiaolong Yang, School of Communication and Information Engineering, Chongqing University of Posts and Telecommunications, Chongqing, China.

Wei Nie, School of Communication and Information Engineering, Chongqing University of Posts and

Telecommunications, Chongqing, China.

Liangbo Xie, School of Communication and Information Engineering, Chongqing University of Posts and Telecommunications, Chongqing, China.

\section{Funding}

This work was supported in part by the Science and Technology Research Program of Chongqing Municipal Education Commission (KJZD-K202000605, KJQN202000630, KJQN201900603), the Chongqing Natural Science Foundation Project (cstc2020jcyj-msxmX0842, cstc2020jcyj-msxmX0865), and the National Natural Science Foundation of China (61901076).

Availability of data and materials

All data generated or analyzed during this study are included in this paper.

\section{Declarations}

\section{Ethics approval and consent to participate}

This paper does not contain any studies with human participants or animals performed. All data and procedures performed in this paper were in accordance with the ethical standards of research community.

\section{Consent for publication}

Not applicable. 


\section{Competing interests}

The authors declare that they have no competing interests.

\section{Received: 5 February 2021 Accepted: 26 April 2021}

Published online: 17 May 2021

\section{References}

1. A. Blanco, N. Ludant, P. J. Mateo, Z. Shi, Y. Wang, J. Widmer, in Proceedings of the Personal, Indoor and Mobile Radio Communications (PIMRC), Performance Evaluation of Single Base Station ToA-AoA Localization in an LTE Testbed (IEEE, Istanbul, 2019), pp. 1-6

2. K. Kitao, T. Imai, M. Inomata, in Proceedings of the Wireless Communications and Networking Conference (WCNC), Study on Path Identification at $40 \mathrm{GHz}$ Band in NLOS Urban Street Cell Environment (IEEE, Marrakesh, 2019), pp. 1-4

3. M. Zhou, Y. Wang, Z. Tian, Y. Lian, Y. Wang, B. Wang, Calibrated data simplification for energy-efficient location sensing in internet of things. IEEE Internet Things J. 6(4), 6125-6133 (2019)

4. M. Zhou, Y. Wang, Y. Liu, Z. Tian, An information-theoretic view of WLAN localization error bound in GPS-denied environment. IEEE Trans. Veh. Technol. 68(4), 4089-4093 (2019)

5. J. H. Reed, K. J. Krizman, B. D. Woerner, T. S. Rappaport, An overview of the challenges and progress in meeting the E-911 requirement for location service. IEEE Commun. Mag. 36(4), 30-37 (1998)

6. N. Garcia, H. Wymeersch, E. G. Larsson, A. M. Haimovich, M. Coulon, Direct localization for massive MIMO. IEEE Trans. Signal Process. 65, 2475-2487 (2017)

7. S. Wu, S. Zhang, D. Huang, A TOA-based localization algorithm with simultaneous NLOS mitigation and synchronization error elimination. IEEE Sensors Lett. 3, 1-4 (2019)

8. Y. T. Chan, K. C. Ho, A simple and efficient estimator for hyperbolic location. IEEE Trans. Signal Process. 42(8), 1905-1915 (1994)

9. J. J. Caffery, in Proceedings of the Vehicular Technology Conference Fall 2000, A new approach to the geometry of TOA location (IEEE, Boston, 2000), pp. 1943-1949

10. C. Li, S. Zhang, P. Liu, F. Sun, J. M. Cioffi, L. Yang, Overhearing protocol design exploiting inter-cell interference in cooperative green networks. IEEE Trans. Veh. Technol. 65, 441-446 (2016)

11. C. Li, P. Liu, C. Zou, F. Sun, J. M. Cioffi, L. Yang, S.pectral.-e.icientc.ellular.c.ommunications.w.ith.c.oexistent. one-, t.wo.-h.op. transmissions. IEEE Trans. Veh. Technol. 65, 6765-6772 (2016)

12. J. Su, R. Xu, S. Yu, B. Wang, J. Wang, Redundant rule detection for software-defined networking. KSII Trans. Internet Inform Syst. 14, 2735-2751 (2020)

13. M. Zhou, X. Li, Y. Wang, S. Li, Y. Ding, W. Nie, 6 G multi-source information fusion based indoor positioning via Gaussian kernel density estimation. IEEE Internet Things J. 2020, 1-1 (2020)

14. C. Li, F. Sun, J. M. Cioffi, L. Yang, M. I. M. O. Energy efficient, relay transmissions via joint power allocations. IEEE Trans. Circ. Syst. 61, 531-535 (2014)

15. C. Li, H. J. Yang, F. Sun, J. M. Cioffi, L. Yang, Multiuser overhearing for cooperative two-way multiantenna relays. IEEE Trans. Veh. Technol. 65, 3796-3802 (2016)

16. C. Li, H. J. Yang, F. Sun, J. M. Cioffi, L. Yang, Cioffi, Adaptive overhearing in two-way multi-antenna relay channels. IEEE Signal Process. Lett. 23,117-120 (2016)

17. M. P. Wylie, J. Holtzman, in Proceedings of the International Conference on Universal Personal Communications, The non-line of sight problem in mobile location estimation (IEEE, Cambridge, 1996), pp. 827-831

18. J. Su, R. Xu, S. Yu, B. Wang, J. Wang, Idle slots skipped mechanism based tag identification algorithm with enhanced collision detection. KSIII Trans. Internet Inform. Syst. 14, 2294-2309 (2020)

19. J. Zhang, Y. Zhuo, Y. Zhao, in Proceedings of the International Conference on Information, Networking and Automation (ICINA), Mobile location based on SVM in MIMO communication systems (IEEE, Kunming, 2010), pp. 360-363

20. M. Porretta, P. Nepa, G. Manara, F. Giannetti, M. Dohler, B. Allen, A. H. Aghvami, A novel single base station location technique for microcellular wireless networks: description and validation by a deterministic propagation model. IEEE Trans. Veh. Technol. 53, 1502-1514 (2004)

21. M. Zhaounia, M. A. Landolsi, R. Bouallegue, Mobile localization under non-line-of-sight conditions using scattering information. Int. J. Wirel. Inform. Netw. 17(1-2), 1-10 (2010)

22. S. Al-Jazzar, J. Caffery, H. You, Scattering-model-based methods for TOA location in NLOS environments. IEEE Trans. Veh. Technol. 56, 583-593 (2007)

23. A. Borhani, M. Patzold, A unified disk scattering model its angle-of-departure and time-of-arrival statistics. IEEE Trans. Veh. Technol. 62, 473-485 (2013)

24. S. Al-Jazzar, J. Caffery, in Proceedings of the Vehicular Technology Conference, ML and Bayesian TOA location estimators for NLOS environments (IEEE, Vancouver, 2002), pp. 1178-1181

25. D. Fan, L. Jin, K. Huang, in Proceedings of the IEEE Global Telecommunications Conference, Pseudo Target Dynamic Feasible Region Constraint Location Method Using Single Observer in NLOS Environment (IEEE, New Orleans, 2008), pp. 1-5

26. S. T. Yoon, K. S. Shim, K-means clustering for handling high-dimensional large data. J. KIISE Comput. Pract. Lett. 18, 55-59 (2012)

27. I. Leichter, M. Lindenbaum, E. Rivlin, Mean shift tracking with multiple reference color histograms. Comput. Vision Image Underst. 114, 400-408 (2010)

\section{Publisher's Note}

Springer Nature remains neutral with regard to jurisdictional claims in published maps and institutional affiliations. 